\title{
KNOWLEDGE ABOUT COELIAC DISEASE: RESULTS OF SURVEY CONDUCTED AMONG PERSONS SCREENED USING A SELF- ADMINISTERED TRANSGLUTAMINASE-BASED TEST
}

\author{
N. Drabińska, N. Bączek and U. Krupa-KozaK* \\ Department of Chemistry and Biodynamic of Food, \\ Institute of Animal Reproduction and Food Research of the Polish Academy of Sciences \\ Tuwima Str, 10, 10-747 Olsztyn. Poland
}

(Received: 15 May 2015; accepted: 16 September 2015)

\begin{abstract}
Coeliac disease (CD) is an autoimmune, gluten-related disorder occurring in genetically predisposed individuals. The keystone to CD management is a gluten-free diet (GFD). Recently, media have been promoting the application of a GFD, however, this is necessary only in gluten-related disorders. The aim of the present study was to evaluate the knowledge on CD among individuals, who conducted a self-administered coeliac disease test. Three hundred adult volunteers took part both in the anti-tissue transglutaminase screening and the survey concerning knowledge on $\mathrm{CD}$. Five positive $\mathrm{CD}$ tests were obtained, representing $1.67 \%$ of the analysed population. In general, the questionnaire respondents were familiar with the issue of $\mathrm{CD}$. The majority of them correctly defined CD as gluten intolerance, and realized that it may occur at any age. Gastrointestinal problems were easily associated with CD, however, extraintestinal symptoms were less frequently recognized as a manifestation of this disease. A GFD was properly identified as a method of treating CD by $95 \%$ of the respondents. Self-administered, transglutaminasebased screened persons presented a good level of knowledge on $\mathrm{CD}$, regardless of their gender, place of residence, or education. However, dissemination of knowledge is needed, as CD is still an underestimated problem.

Keywords: coeliac disease, questionnaire, population screening, gluten-free diet, transglutaminase, Poland
\end{abstract}

Coeliac disease (CD) is an autoimmune disease occurring in genetically predisposed individuals. Genetic predisposition is associated with genes encoding the human leukocyte antigen (HLA), known as HLA-DQ2 and HLA-DQ8 (SAPONE et al., 2012). It is manifested by the chronic inflammation of the small intestine, villous atrophy and hyperplastic lesions of the crypts in response to gluten ingestion. Classic, atypical and silent CD can be distinguished. In the classic example of CD, gastrointestinal symptoms, such as chronic diarrhoea, vomiting, and abdominal pain, are observed. Atypical CD, on the other hand, is characterized by minor and/or isolated symptoms (anaemia, osteoporosis, neurological disorders). In silent CD, patients report no symptoms, which contributes to problems with its correct diagnosis (SAPONE et al., 2012). Symptoms of CD may be age-dependent (RomA et al., 2009), and extraintestinal symptoms occur more frequently in older children and adults (GUANDALINI \& Assiri, 2014).

Until recently, the diagnosis of CD was based on the assessment of highly variable clinical status, assays of different specific antibodies, the histological evaluation of intestinal biopsies, and the response to a gluten-free diet (GFD) (GREEN \& CELLIER, 2007). These days, most patients are diagnosed by endoscopy and biopsy following the positive results of serological tests (Mooney et al., 2015). It is recommended to determine the level of anti-

\footnotetext{
* To whom correspondence should be addressed.

Phone: +48 8952346 18; fax: +48 8952401 24; e-mail: u.krupa-kozak@pan.olsztyn.pl
} 
tissue transglutaminase ( $\mathrm{tTG}$ ) antibodies as the first step of $\mathrm{CD}$ diagnosis. Other serological tests, such as anti-endomysial antibodies (EMA) and antibodies against deaminated gliadin peptides (DGP), may also be evaluated in certain cases. A positive result of the abovementioned serological tests allows one to suspect $\mathrm{CD}$, however, for definite diagnosis, the confirmation of a biopsy of the small intestine is needed.

It is estimated that approximately $1 \%$ of the general population suffers from CD (WEST et al., 2003), but a significant percentage of the affected people go unrecognized or are diagnosed incorrectly (FASANO et al., 2003). Therefore, the serological population screening of groups at risk could be of value. Screening the population using conventional serological tests is expensive, difficult to organize, and requires qualified staff and specialized equipment (KorPONAY-SZABÓ et al., 2007). Fortunately, faster methods of detecting antibodies have recently become available. They can be performed on a small blood sample from a finger and do not require the collection of venous blood samples.

Currently, CD has become a popular topic in the media. Information about the disease and portals and forums for patients as well as healthy people who want to learn more about the disease can be accessed easily. The media frenzy propagates a GFD, spreading knowledge concerning gluten and gluten-related disorders and expanding awareness on this subject. On the other hand, media promotes the application of a GFD without reasonable health rationalization. Therefore, it is important to take the available data in critically. The aim of the present study was to evaluate knowledge on CD among individuals who were interested in the topic for personal reasons (relatives of CD patients, individuals suffering from gastrointestinal problems, etc.) and conduct a self-administered transglutaminase-based test to determine how many people may actually be affected.

\section{Materials and methods}

An advertisement concerning $\mathrm{CD}$ testing was published in the local media. Individuals who were interested in the issue of $\mathrm{CD}$ were given the possibility of conducting a self-administered transglutaminase-based test. The interest of the volunteers in the present study resulted mainly from personal motives, such as a relationship with a person suffering from CD, deteriorating health and/or distressing nonspecific symptoms, or just plain curiosity.

\subsection{TTG screening population}

The Local Ethical Committee (Olsztyn, Poland) was informed about the screening of the population for $\mathrm{CD}$. According to them, the studies did not require acceptation of the Committee as a commercial, self-administered immunochromatographic BIOCARD Coeliac Test (Labsystems Diagnostics Oy, Vantaa, Finland) was used in the screening. The test determines the presence of a coeliac-specific IgA anti-tissue transglutaminase antibody together with the total serum IgA in a fresh whole blood sample. All CD screening participants received a free test to perform individually in accordance with the instructions provided by the manufacturer. The possible test results have been presented in Figure 1. All participants were under the supervision of a qualified medical paramedic and covered by insurance from the effects of dangerous accidents. 


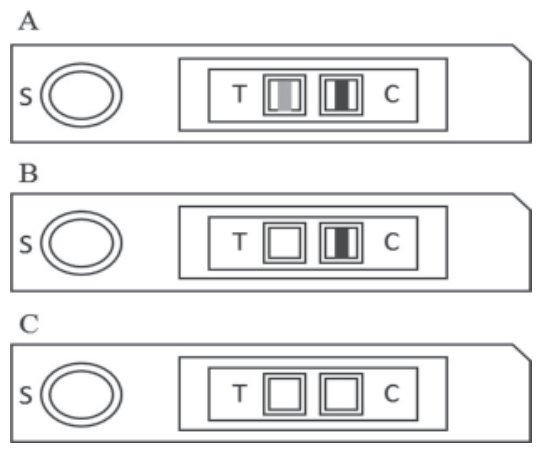

Fig. 1. Possible BIOCARD Coeliac Test results. A: positive test result, a line in the control field (C) and test field (T) appears; B: negative test result, a line appears only in the control field (C); C: invalid test or IgA antibody deficiency.

\subsection{Knowledge on $C D$}

Among the 300 people who took part in the small-scale tTG population screening, a survey concerning their knowledge on CD was carried out. The volunteers signed their informed consent to survey data processing prior to the study. Survey participants were asked to fill out the authors' original questionnaire containing seven multiple-choice questions. Questions concerned mainly the definition, aetiology, symptoms, and treatment of CD. Additionally, the sociodemographic structure of respondents was recorded.

\section{Results and discussion}

\subsection{Population screening results}

Among three hundred BIOCARD coeliac tests performed individually, 5 gave a positive result, which represents $1.67 \%$ of the population. A Hungarian screening study using rapid BIOCARD tests identified 37 newly diagnosed cases of CD among children in a cohort of 2690 (KorPONAY-SzABÓ et al., 2007). Another study using this test allowed 117 CD persons to be identified out of 121 untreated CD patients, which gave results similar to standard serological tests (RAIVIO et al., 2006). Nowadays, it is assumed that anywhere from 0.5 to $2 \%$ of the global population may suffer from CD (REwers, 2005; GuANDALini \& Assiri, 2014), including $0.3-1.0 \%$ of the European population or those with European ancestry (MEARIN et al., 2005). Therefore, it is important to find a quick and cheap way of carrying out mass CD screening, especially in groups of increased risk. Although the presence of anti-tTG antibodies does not definitely mean $C D$, the results obtained from the present study may suggest a higher then suspected prevalence of CD within the analysed population. Each participant with a positive test result was encouraged to consult a gastroenterologist and obtained information about gastroenterological clinics helpful in further diagnosis. 


\subsection{Knowledge on $C D$}

Of the three hundred filled out questionnaires obtained, twenty-two (7\%) were rejected as the responders did not declare their socio-demographic status, or provided incomplete information. The remaining 278 questionnaires were analysed. The socio-demographic structure of the survey participants is presented in Table 1.

\begin{tabular}{|c|c|}
\hline \multicolumn{2}{|l|}{ Survey participants: } \\
\hline Female (N, \%) & 188,68 \\
\hline Male (N, \%) & 90,32 \\
\hline $\mathrm{F} / \mathrm{M}$ ratio & $1: 0.48$ \\
\hline \multicolumn{2}{|l|}{ Age (years): } \\
\hline 18-29 (N, \%) & 103,37 \\
\hline 30-39 (N, \%) & 78,28 \\
\hline 40--49 (N, \%) & 54,19 \\
\hline$>50(\mathrm{~N}, \%)$ & 43,15 \\
\hline \multicolumn{2}{|l|}{ Education: } \\
\hline Primary (N, \%) & 24,9 \\
\hline Secondary (N, \%) & 75,27 \\
\hline Higher (N, \%) & 179,64 \\
\hline \multicolumn{2}{|l|}{ Place of residence: } \\
\hline Olsztyn city (N, \%) & 205,74 \\
\hline Small city (N, \%) & 30,11 \\
\hline Village (N, \%) & 43,15 \\
\hline
\end{tabular}

The first survey question diversified the participants into a dominant group of respondents who had heard of the term "coeliac disease" ( $70 \%$; among them $78 \%$ women and $56 \%$ men) and were asked to answer the remaining questions, and those who had not (30\%) and did not continue filling out the questionnaire. The next question defined CD. Nearly all of the respondents (94\%) correctly defined CD as gluten intolerance; among them, 26 individuals described $\mathrm{CD}$ as both gluten intolerance and the chronic inflammation of the small intestine (Fig. 2A). In the question concerning the age of persons suffering from $\mathrm{CD}$, most respondents (nearly $90 \%$ ) answered that children, adolescents, and adults alike may suffer from the disease (Fig. 2B). Until recently, CD was misinterpreted as a "rare disease of young children, which they grow out of after a few years of sticking to a proper diet". This myth of CD being a childhood disease continues to be deeply maintained, even in the medical community. The last multiple-answer question concerned the symptoms of CD (Fig. 2C). Although all the possible answers were correct, only a few of the respondents ( 29 individuals) indicated all the possibilities. In general, gastrointestinal problems were easily associated with CD (87\%), whereas atypical symptoms, such as constant fatigue, bone and joint pain, and anaemia were not always seen as signs of $\mathrm{CD}$. The highly variable clinical picture of $\mathrm{CD}$ complicates its accurate diagnosis. Patients touched with CD often complain of frank malnutrition, which is 
a consequence of the malabsorption of several nutrients and vitamins; anaemia and iron deficiency are frequent hematologic manifestations of CD (HARPER et al., 2007). Calcium deficiency in patients suffering from $\mathrm{CD}$ affects the reduction of bone mineral density, leading to osteopenia and osteoporosis (KruPa-KozaK, 2014; Grace-Farfaglia, 2015). Endocrine (short stature, delayed puberty) and reproductive disorders (miscarriages, infertility) are also observed among people with CD (RIVERA et al., 2013). Therefore, familiarity with the wide spectrum of CD symptoms is crucial to its proper diagnosis.

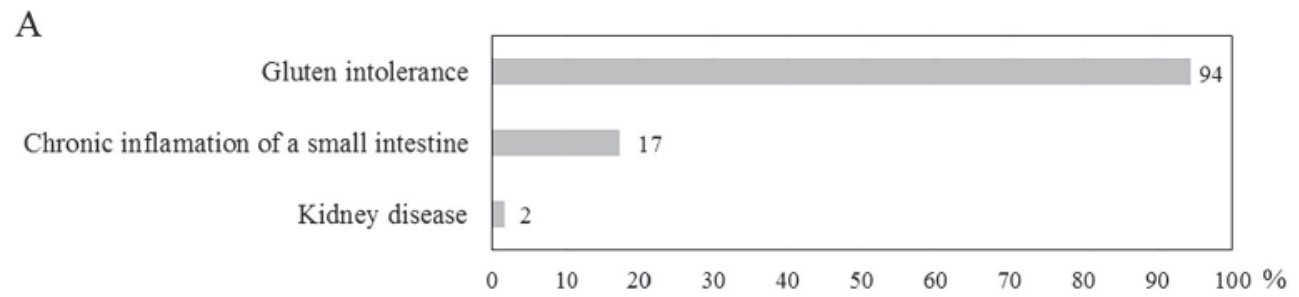

B

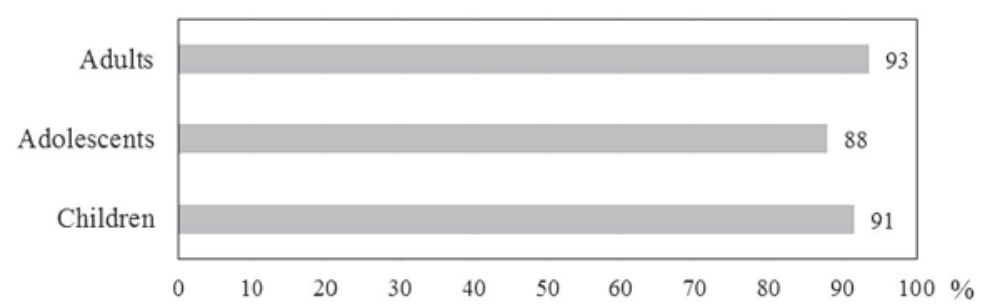

$\mathrm{C}$

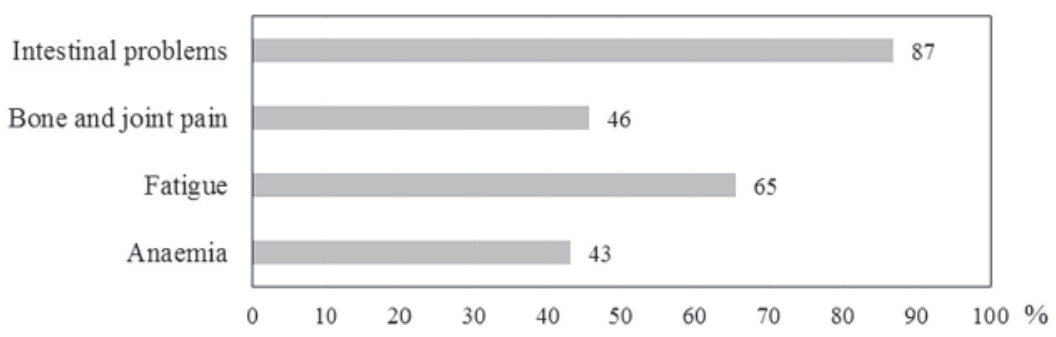

Fig. 2. Answers of respondents participating in the survey for multiple-answer questions: A: "What is coeliac disease?"; B: "Who can suffer from coeliac disease"; C: "What are the symptoms of CD?"

The answers of survey participants to the single-answer questions are presented in Figure 3. Gluten was correctly identified as the factor responsible for $\mathrm{CD}$ development by the majority of responders (Fig. 3A). Only $4 \%$ of those interviewed pointed to other triggers, such as milk proteins and peanuts, which were regarded as incorrect answers. The genetic predisposition to $\mathrm{CD}$ was the issue tested in the next question. More than half of the questionnaire participants realized that the $\mathrm{CD}$ may develop in genetically predisposed individuals (Fig. 3B). The third question verified the respondents' knowledge concerning the methods of treating $\mathrm{CD}$. The greater part of the interviewed persons correctly selected a GFD as the method of treating CD (Fig. 3C). However, 5\% of them answered the question differently, additionally indicating a diet of easily digestible foods, surgical intervention, or 
vitamin $\mathrm{C}$ as methods of treating the disease. Currently, the only treatment for CD scientifically proven to be effective is the strict and lifelong exclusion of gluten from one's diet. It is generally accepted that in a GFD, all foods and medications containing gluten from wheat, rye, and barley, and their derivatives must be eliminated, as even small quantities of gluten may be harmful. The complete removal of gluten from the diet of patients suffering from $\mathrm{CD}$ results in symptomatic, serologic, and histological remission in a majority of them (FASANO \& CATASSI, 2001; PietZaK, 2005). In children with CD, growth and development returns to normal with adherence to a GFD, whereas in adults, many disease complications can be avoided.

A

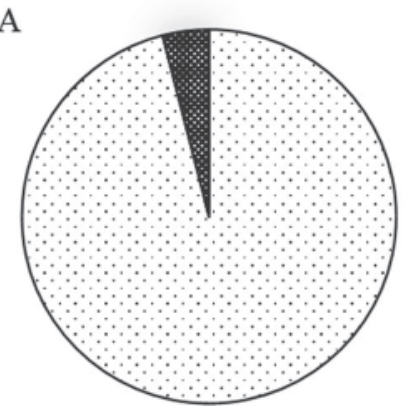

B

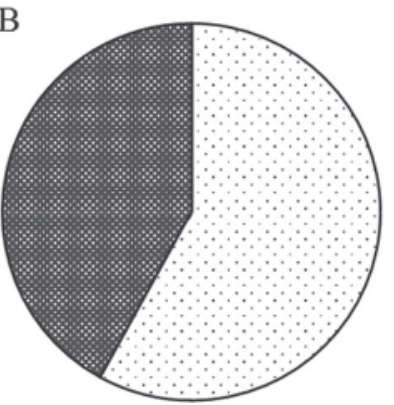

$\mathrm{C}$

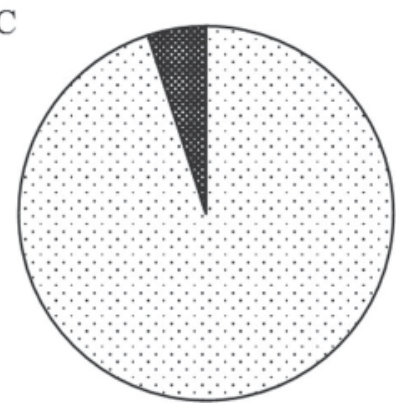

Fig. 3. Answers of respondents participating in the survey to single-answer questions: A: "What it the environmental trigger of CD?”: : $:$ : Gluten; ․․․: Other answers.B: „Is CD genetically determined?”: :

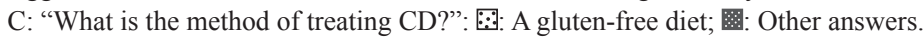

\section{Conclusions}

Based on the results obtained, it can be stated that, in general, self-administered tTG screened persons presented a good level of knowledge on $\mathrm{CD}$, regardless of their gender, place of residence, or education. The majority of them correctly defined $\mathrm{CD}$ as gluten intolerance and realized that it may occur at any age. Gastrointestinal problems were easily associated with $\mathrm{CD}$, however, extra-intestinal symptoms were less frequently recognized as a manifestation of the disease. Wherefore, appropriate health education, effective campaigns, and continuous popularization of the knowledge concerning the prevalence, symptoms, and treatment of $\mathrm{CD}$ among the Polish population is very important, as CD, particularly in its atypical and silent form, is still an underestimated problem.

The research was funded by funds of FUSION NIGHT in the frame of European Researchers Night 2014 in Olsztyn. The authors would like to thank all volunteers who participated in the study. The authors declare that they have no conflict of interests.

\section{References}

Fasano, A., Berti, I., Gerarduzzi, T., Not, T., Colletti, R.B., Drago, S., Elitsur, Y., Green, P.H., Guandalini, S., Hill, I.D., Pietzak, M., Ventura, A., Thorpe, M., Kryszak, D., Fornaroli, F., Wasserman, S.S., Murray, J.A. \& Horvath, K. (2003): Prevalence of coeliac disease in at-risk and not-at-risk groups in the United States: A large multicenter study. Arch. Intern. Med., 163, 286-292. 
Fasano, A. \& CAtassi, C. (2001): Current approaches to diagnosis and treatment of celiac disease: an evolving spectrum. Gastroenterology, 120, 636-651.

Green, P.H. \& Cellier, C. (2007): Celiac disease. New Engl. J. Med., 357(17), 1731-1743.

Grace-Farfaglia P. (2015): Bones of contention: Bone mineral density recovery in celiac disease - A systematic review. Nutrients, 7, 3347-3369.

Guandalini, S. \& Assiri, A. (2014): Celiac disease: a review. JAMA Pediatr., 168, 272-278.

Harper, J.W., Holleran, S.F., Ramakrishnan, R., Bhagat, G. \& Green, P.H. (2007): Anemia in celiac disease is multifactorial in etiology. Am. J. Hematol., 82, 996-1000.

Korponay-Szabó, I.R., Szabados, K., Pusztai, J., Uhrin, K., Ludmány, E., Nemes, É., Kaukinen, K., Kapitány, A., Koskinen, L., Sipka, S., ImRe, A. \& MÄKI, M. (2007): Population screening for coeliac disease in primary care by district nurses using a rapid antibody test: diagnostic accuracy and feasibility study. BMJ, 335, 1244.

Krupa-Kozak, U. (2014): Pathologic bone alterations in celiac disease: Etiology, epidemiology, and treatment. Nutrition, 30, 16-24.

Mearin, M.L., Ivarsson, A. \& Dickey, W. (2005): Coeliac disease: is it time for mass screening? Best Pract. Res. Cl. Ga., 19(3), 441-452.

Mooney, P.D., Wong, S.H., Johnston, A.J., Kurien, M., Avgerinos, A. \& Sanders, D.S. (2015): Increased detection of celiac disease with measurement of deamidated gliadin peptide antibody before endoscopy. Clin. Gastroenterol. H., 13(7), 1278-1284. doi:10.1016/j.cgh.2015.01.010.

Pietzak, M.M. (2005): Follow-up of patients with celiac disease: achieving compliance with treatment. Gastroenterology, 128, 135-141.

Raivio, T., Kaukinen, K., Nemes, E., Laurila, K., Collin, P., Kovács, J.B., Mäki, M. \& Korponay-Szabó, I.R. (2006): Self transglutaminase-based rapid coeliac disease antibody detection by a lateral flow method. Aliment. Pharm. Ther., 24(1), 147-154.

Rewers, M. (2005): Epidemiology of celiac disease: what are the prevalence, incidence, and progression of celiac disease? Gastroenterology, 128, 47-51.

Rivera, E., Assiri, A. \& Guandalini, S. (2013): Celiac disease. Oral Dis., 19, 635-641.

Roma, E., Panayiotou, J., Karantana, H., Constantinidou, C., Siakavellas, S.I., Krini, M., Syriopoulou, V.P. \& Bamias, G. (2009): Changing pattern in the clinical presentation of pediatric celiac disease: a 30-year study. Digestion, 80, 185-191.

Sapone, A., Bai, J., Ciacci, C. Dolinsek, J., Green, P.H., Hadjivassiliou, M., Kaukinen, K., Rostami, K., Sanders, D.S., Schumann, M., Ullrich, R., Villalta, D., Volta, U., Catassi, C. \& Fasano, A. (2012): Spectrum of gluten-related disorders: consensus on new nomenclature and classification. BMC Med., 10, 13.

West, J., Logan, R.F., Hill, P.G. Lloyd, A., Lewis, S., Hubbard, R., Reader, R., Holmes, G.K. \& Khaw, K.T. (2003): Seroprevalence, correlates, and characteristics of undetected coeliac disease in England. Gut, 52, 960-965. 